\title{
APPLICATION OF ANT COLONY OPTIMISATION IN DISTRIBUTION TRANSFORMER SIZING
}

\author{
O. Folorunso ${ }^{1}$, P. A. Oriaifo ${ }^{2,}{ }^{*}$, N. S. Idiagi ${ }^{3}$ and E. A. Ogujor ${ }^{4}$ \\ 1, 2, 3,4, Department of EleCtrical/Electronic EnGR'G., University of BEnin, BEnin City, Edo State, NigERIA \\ E-mail addresses: ${ }^{1}$ folorunsoo@abuad.edu.ng, ${ }^{2}$ patrick.oriaifo@uniben.edu, ${ }^{3}$ neville.idiagi@uniben.edu, \\ 4 emma.ogujor@uniben.edu.ng
}

\begin{abstract}
This study proposes an optimisation method for transformer sizing in power system using ant colony optimisation and a verification of the process by MATLAB software. The aim is to address the issue of transformer sizing which is a major challenge affecting its effective performance, longevity, huge capital cost and power loss. This method accounts for the constraints imposed by the load capacity and the thermal overload that the transformer serves throughout its lifetime. The objective function to be minimised includes the transformer capital cost as well as the energy loss cost. In this paper, the Optimal Transformer Sizing (OTS) problem which is fundamentally the basic routine for the location of transformer was addressed by means of the heuristic Ant System Method using the Elitist strategy, called Elitist Any System (EAS). EAS belong to the family of Ant Colony Optimisation (ACO) algorithm. ACO when appropriately applied determines the least cost path, taking into consideration the various essential factors including transformer bid price, growth rate, inflation rate, peak load, thermal deviation and energy loss cost. The study demonstrated a significant saving in capital cost using this approach as evidenced from the changes to the transformer following the initial installed capacity of $190 \mathrm{kVA}$ to $320 \mathrm{kVA}$ in the second stage and then finally to $630 \mathrm{kVA}$ in the third stage which effectively supported the remaining period under consideration. This finding is in contrast to the traditional simplified sizing strategy usually adopted by utilities companies.
\end{abstract}

Keywords: ant colony, optimization, transformer sizing, distribution transformer

\section{INTRODUCTION}

A transformer is a device that changes ac electric power at one frequency and voltage level to ac electrical power at the same frequency and another voltage level through the action of a magnetic field [2]. Whilst in service, there has been a surge in transformer breakdown arising mainly from poor sizing and undermining population growth leading to overload in transformer. The optimal transformer sizing problem which is synonymous to transformer location involves finding the proper transformer capacities and technical characteristics so that the overall cost of the transformer over its life cycle can be minimised and the peak loading condition can be met [3].

Most electrical power machines are designed to operate using a specific frequency and voltage. The problem with this seemingly elementary concept is that voltage and frequency does vary from country to country and even sometimes by localities or areas within a country. These two parameters can be controlled so that it poses little challenge to power system. However, other parameters such as temperature and contingency like loading may not be easily controlled.

With the rate of population growth constantly increasing in Nigeria, the demand for electrical power will significantly increase to meet current demand. Therefore, if proper future analysis is not taken into consideration, the problem of epileptic power (cut in electricity) would persist and continue to be the order of the day. Thus, the major challenges facing the transformer and its life span include improper location of transformer, sizing and loading effect [3]. An approach towards addressing these problems will involve proper optimisation by considering the initial loading condition of the transformer future expected load, temperature rise, the transformer's iron and copper losses, etc.

Transformer sizing optimisation is a multi-objective function, and therefore the optimal choice of the transformer size cannot be directly achieved. Generally, the strategy adopted by the electric utility companies 
for transformer sizing is to install transformers which meet the current load demand for a specific location without allowance for the more demand in the future. Consequently, the future demand of required power from the existing installed transformer become insufficient in meeting the demand of the growing population. This approach used by utility companies in Nigeria seems obsolete with lots of uncertainty for the future. One method referred to as Deterministic Optimisation methods could offer solution to this problem by applying either dynamic programming [3] or integer programming [11]. However, the wide spectrum of transformer sizes and various load types involved in the electric utility distribution system make the transformer sizing a challenging combinatorial optimisation problem, since the space of solutions is huge. That is why stochastic optimisation methods may prove to provide more robust solutions [8].

Ant Colony Optimisation (ACO) algorithm was created taking into account the various objective functions using Elitist Ant System (EAS) approach for the optimisation of the transformer sizing problem. Dorigo [4] first proposed the EAS in his doctoral research and later on in a peer reviewed publication [5]. The ACO algorithm is inspired by the behaviour of real ant colonies used to solve combinatorial optimisation problem. The real ants lay down in some quantity an aromatic substance, known as pheromone, on their way to food source.

The pheromone quantity depends on the length of the path and the quality of the discovered food source [12]. An ant chooses an exact path in connection with the intensity of the pheromone. The pheromone trail evaporates over time if no more pheromone is laid down. Other ants are attracted to follow the pheromone trail. Therefore, the path will be marked again and it will attract more ants to use the same path. The pheromone trail on paths leading to rich food sources closest to the nest will be more visited and will therefore grow faster. In this way, the best solution has more intensive pheromone and higher probability to be chosen [12].

In this study, an ant colony optimisation technique was applied to transformer sizing problem (generally threephase, oil immersed, air cooled transformers). The objective function to be minimised include transformer capital cost and energy loss cost. The calculation takes into account constraint such as insulation aging throughout the transformer life cycle for the specified period of installation.

Although, there is extensive literature on transformer sizing problem, very few explained the dynamics of transformer sizing using MATLAB software to compute the optimisation algorithm. As MATLAB software has the capability to handle extensive data and produce repeatable results of cumbersome analysis with much less cost, it was thought beneficial to simulate an optimisation using the software to advance the EAS model. Elefterios, et al [8] proposed the MATLAB simulation that was based on the non-deterministic methodology.

\section{ANALYSIS OF PARAMETERS REQUIRED FOR OPTIMAL TRANSFORMER SIZING}

This section summarises an example from Elefterios et al, 2007 to illustrate the application of ACO algorithm in the optimal sizing of a distribution transformer. The analysis includes forecasting of the transformer life span, transformer thermal loading, calculation of transformer energy loss cost, overloading capacity, and insulation ageing.

Transformer loading and temperature differential determines the life span of every transformer. Consequently, it is expected to forecast the future demand of the area for a specific period of time with respect to demand rate.

Stochastic/probabilistic extrapolation method is a very good method for determining the future demand of electrical quantities having known the initial demand and its consequent loading. The least square method is an example and the most frequently used method adopted.

In calculating the transformer thermal loading, the guidelines imposed by the IEEE Standard C57.91-1995 (IEEE Guide for Loading Mineral Oil-Immersed Transformers, IEEE StC57.91, 2002) is implemented. The mathematical model used in these calculations is described below:

The top-oil temperature rise, $\Delta \varphi_{T O}$, at a time after a step load change is given by:

$$
\begin{gathered}
\Delta \varphi_{T O}=\left(\Delta \varphi_{T O}, u-\Delta \varphi_{T O}, i\right) \cdot\left\lfloor 1-e^{\left.\frac{-1}{\tau T O}\right\rfloor}+\Delta \varphi_{T O}, i\right. \\
\Delta \varphi_{T O}, U=\Delta \varphi_{T O}, R \cdot\left(\frac{K^{2} \cdot R+1}{R+1}\right)^{n} \\
\Delta \varphi_{H}=\left(\Delta \varphi_{H}, u-\Delta \varphi_{H}, i\right) \cdot\left\lfloor 1-e^{\frac{-1}{\tau w}}+\Delta \varphi_{H}, i\right. \\
\Delta \varphi_{H}=\Delta \varphi_{H}, U
\end{gathered}
$$

In (1) to (4), $\Delta \varphi_{T O}, i$, and $\Delta \varphi_{T O}, U$, are the initial and ultimate top-oil rise over ambient temperature during the considered time period, $\tau T O$ is the oil time constant (in hours) for the considered load. Also, $\Delta \varphi_{H}, i$, and $\Delta \varphi_{H}, u$,represent the initial and ultimate winding hottest spot temperature rise over top-oil temperature during the considered time period, $\tau w$ stands for the winding time constant at hot spot location (in hours) and $\Delta \varphi_{T O}, R$, the top-oil rise over ambient temperature at rated load.

The ultimate winding hottest spot temperature rise over top-oil temperature is given by (5) and (6):

$$
\Delta \varphi_{H}, U=\Delta \varphi_{H}, R \cdot K^{2 n}
$$




$$
\varphi_{H}=\varphi_{A}+\Delta \varphi_{T O}+\Delta \varphi_{H}
$$

For insulation ageing, there is a potential risk to the effective performance of any transformer operating at a temperature above $140^{\circ} \mathrm{C}$ as suggested by the IEEE standard. For the purpose of calculation of transformer loading limits, this value is not taken as the maximum. This is because thermal ageing is a cumulative process. For simulation purposes, a maximum hot-spot temperature of $120^{\circ} \mathrm{C}$ may be chosen, based on the relative ageing rate of the insulation in the transformer [10]. In order to determine the transformer loading limits, the calculation of the hottest spot temperature is repeated for each year of the study period, on an hourly basis, according to the daily load curve.

$$
K_{t}^{j}=K_{t}^{0} \cdot(1+r)^{j}
$$

Here, $K_{t}^{j}$, the per unit load of hour $t$ at year $j$ of the study, $K_{t}^{0}$ the per unit load of hour $t$ at year 0 and $r$, yearly load growth rate. The calculation of transformer energy loss cost $\left(E_{N L L}^{k}\right)$ is calculated according to eqns (8) and (9):

$$
\begin{aligned}
E_{N L L}^{k} & =N L L . H P Y . k \\
E_{L L}^{k} & =L L . l f^{2} . H P Y . k
\end{aligned}
$$

Here, HPY is the number of hours per year, equal to 8760 and If is the load factor, i.e. the mean transformer loading over its lifetime. The cost of total energy corresponding to the transformer $N L L$ for the period of kyears $C_{N L L}^{k}$ (in naira) and the cost of energy corresponding to the transformer $L L$ over a period of kyears $C_{L L}^{k}$ (in naira) are derived as follows:

$$
\begin{gathered}
C_{N L L}^{k}=E_{N L L}^{k} \cdot C Y E C \\
C_{N L L}^{k}=E_{L L}^{k} \cdot C Y E C
\end{gathered}
$$

Finally, the total cost of the transformer energy loss $E_{L}^{k}$ for the period of $k$ years of the study is given by (12) and is the objective function to be minimized:

$$
E_{L}^{k}=C_{N L L}^{k}+C_{L L}^{k}
$$

\section{ELITIST ANT SYSTEM METHOD}

The operational mode of EAS algorithm is as follows: the artificial ants of the colony move concurrently and asynchronously, through adjacent states of a problem, which can be represented in the form of a weighed graph. The movement is made according to a transition rule, called random proportional rule, through a stochastic mechanism. When ant $k$ is in node $i$ and has so far constructed the partial solutions ${ }^{p}$, the probability of going to node $j$ is given by Marinaet al, [9]:

$$
p_{i j}^{k}=\left\{\begin{array}{c}
\frac{\tau_{i j}^{\alpha}+n_{i j}^{\beta}}{\sum \tau_{i j}^{\alpha}+n_{i j}^{\beta},} \text { if } c_{i j} \in N\left(s^{p}\right) \\
c_{i j} \in N\left(s^{p}\right) \\
0, \quad \text { otherwise }
\end{array}\right.
$$

In (13), $N\left(s^{p}\right)$ is the set of feasible nodes when being in node $i$, i.e. edged $(i, I)$ where $l$ is the node not yet visited by the ant $k$. The parameter $\alpha$ and $\beta$ controls the relative importance of the pheromone versus the heuristic information value $n_{i j}$, given by:

$$
n_{i j}=\frac{1}{d_{i j}}
$$

In (14), $d_{i j}$ is the weight of each edge. Regarding parameters $\alpha$ and $\beta$, their roles are as follows; if $\alpha=$ 0 , those nodes with better heuristic preference have a higher probability of being selected. However, if $\beta=0$, only the pheromone trails are considered to guide the constructive process, which can cause a quick stagnation, i.e. a situation where the pheromone trails associated with some transitions are significantly higher than the remainder, thus making the ants build the same solutions. Hence, there is a need to establish a proper balance between the importance of heuristic and pheromone trail information.

The main characteristic of the EAS technique is that (at each iteration) the pheromone values are updated by all the $k$ ants that built a solution in the iteration itself. The pheromone $\tau_{i j}$, associated with the edge joining nodes $i$ and $j$, is updated as follows [1]:

$$
\tau_{i j}=(1-\rho) \cdot \tau_{i j}+\sum_{m=1}^{k}\left(\Delta \tau_{i j}^{k}+\varepsilon \tau_{i j}^{e l i t e}\right)
$$

Here $\rho \epsilon(0,1)$ the evaporation rate, $k$ is the number of ants, $\varepsilon$ is the number of elitist ants, and $\Delta \tau_{i j}^{k}$ is the quantity of pheromone laid on edge $(i, j)$ by ant $k$, calculated as follows:

$$
\Delta \tau_{i j}^{k}=\left\{\begin{array}{lr}
\frac{Q}{L_{k}}, & \text { if ant } k \text { used edge }(i, j) \text { in its tour } \\
0, & \text { otherwise }
\end{array}\right.
$$

In (16) $\mathrm{Q}$ is a constant for pheromone update, and $L_{k}$ is the length (or the weight of the edge) of the tour constructed by ant $k$. Furthermore, shorter paths will tend to have higher pheromone densities than longer paths since pheromone density decreases over time due to evaporation [6]. This shortest path represents the global optimal solution and all the possible paths represent the feasible region of the problem.

\section{METHODOLOGY}

The steps in the methodology employed in this study are as follows:

(a) Data concerning the transformer to be installed was collected from source. These data consist of bid price, no-load loss, on-load loss and the expected (forecast) load growth rate over the transformer lifetime.

(b) The feasible number of years that each transformer can serve the examined load, based on the thermal calculations was determined. 
(c) The energy loss cost for each transformer for the periods defined by the strategies of the previous step was calculated.

(d) The least cost transformer sizing path was selected utilising EAS. This path corresponds to the optimal transformer sizing strategy.

(e) MATLAB code was developed to validate the Ant Colony Algorithm.

\subsection{Data Collection}

The proposed method is applied for the optimal choice of the transformer size to serve a residential load of initial peak value of $190 \mathrm{kVA}$ with a growth rate of $3.9 \%$ for 30 years, resulting to final peak value of 630 kVA (at the end of the 30th year of the study). Seven ratings are considered, namely 190, 200, 260, 320, 430, 460 and $630 \mathrm{kVA}$. Initially, it is expected to carry out thermal study for the transformers in order to find the exact periods when each transformer would meet the load expectations. The technical parameters of transformers used in this study are shown in Table 1. It can be seen from Figure 1 that there is a linear increase in the sizing of the transformer over the period of study.

Table 1: Technical Parameters of transformers used in this study [7]

\begin{tabular}{cccc}
\hline $\begin{array}{c}\text { Transformer } \\
\text { Size (kVA) }\end{array}$ & $\begin{array}{c}\text { Bid } \\
\text { Price(Naira) }\end{array}$ & $\begin{array}{c}\text { No-Load } \\
\text { Losses } \\
(\mathrm{kW})\end{array}$ & $\begin{array}{c}\text { On-Load } \\
\text { Losses } \\
(\mathrm{kW})\end{array}$ \\
\hline 190 & 1100000 & 3.1 & 14.0 \\
200 & 1200000 & 3.6 & 17.5 \\
260 & 1300000 & 4.1 & 22.0 \\
320 & 1450000 & 4.6 & 27.0 \\
430 & 1500000 & 11.8 & 63.0 \\
460 & 1680000 & 15.0 & 74.0 \\
630 & 3360000 & 15.4 & 63.1 \\
\hline
\end{tabular}

\subsection{Application of Ant Colony Optimisation Algorithm to Transformer Sizing Problem}

The application of ant colony algorithm to the optimal sizing of the transformers with technical parameters shown in Table 1, is achieved by applying (1) to (12) repeated for the seven transformers and each year of the study, resulting to time period that the transformer would be in operation, (i.e. years that the transformer is able to withstand the respective thermal loading). These periods were used to define the stages of the graph of Figure 2. In order to define the weight of each arc in the graph of Figure 2, the energy loss cost calculation of each transformer for the studied period was realised. For instance, the arc cost to transit from node 14 to node 21 of Figure 2 is computed as follows: given that $\mathrm{NLL}=15.4 \mathrm{~kW}, \mathrm{LL}=63.1 \mathrm{~kW}$, and $\mathrm{HPY}$
$=8760 \mathrm{hr} /$ year and $\mathrm{k}=5$, load factor $l_{f}=0.67$; from eqns (8) and (9), it is determined that:

$$
\begin{array}{r}
E_{N L L}^{5}=15.4 \times 8760=134904 \mathrm{kWh} / \text { year } \\
E_{L L}^{5}=63.1 \times 0.67^{2} \times 8760 \\
=248132.1684 \mathrm{kWh} / \text { year }
\end{array}
$$

Assume that the utility company of Nigeria is selling a unit of electricity (CYEC) for $11.1 \mathrm{Naira} / \mathrm{kWh}$, then from eqns (10) and (11), it is determined that:

$C_{N L L}^{5}=11.1 * 134904=\# 1,497,434.4$ and

$C_{L L}^{5}=11.1 * 248132.1684=\# 2,754,267.069$.

Finally, from eqn. (12) it is determined that:

$$
E_{630}^{5}=\# 4,251,701.469
$$

\subsection{Matlab Result Showing the Optimal Path of the Transformer Size from the Alogorithm Developed}

The execution of the Ant Colony Optimisation algorithm developed for the Optimal Transformer Sizing path yields Figure 3 and its convergence history is shown in Figure 4.

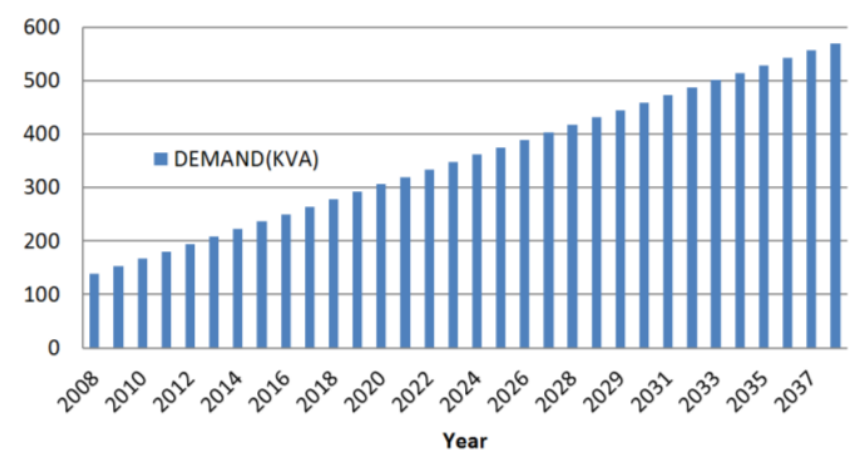

Figure 1: Load forecast for a period of 30 years

\section{DISCUSSION OF RESULTS}

The Ant Colony Optimisation was applied to the transformer sizing problem by considering the size of a distribution transformer to meet the demands of the present and future load to be served. Through detailed calculation of its thermal performance throughout the period the transformer is to serve the located area. The cost function used in the optimisation process includes the transformer energy loss cost as well as its purchasing price. The method was applied for the selection of the optimal size of a distribution transformer to serve a load over a period of 30 years.

The least cost path and the convergence history graphs were obtained as a result of the execution of the MATLAB code to further verify the algorithm (EAS) used. The algorithm showed a better saving cost as shown in Figure3when compared with the traditional simplified sizing strategy usually adopted by utilities companies as shown in Figure 1. 


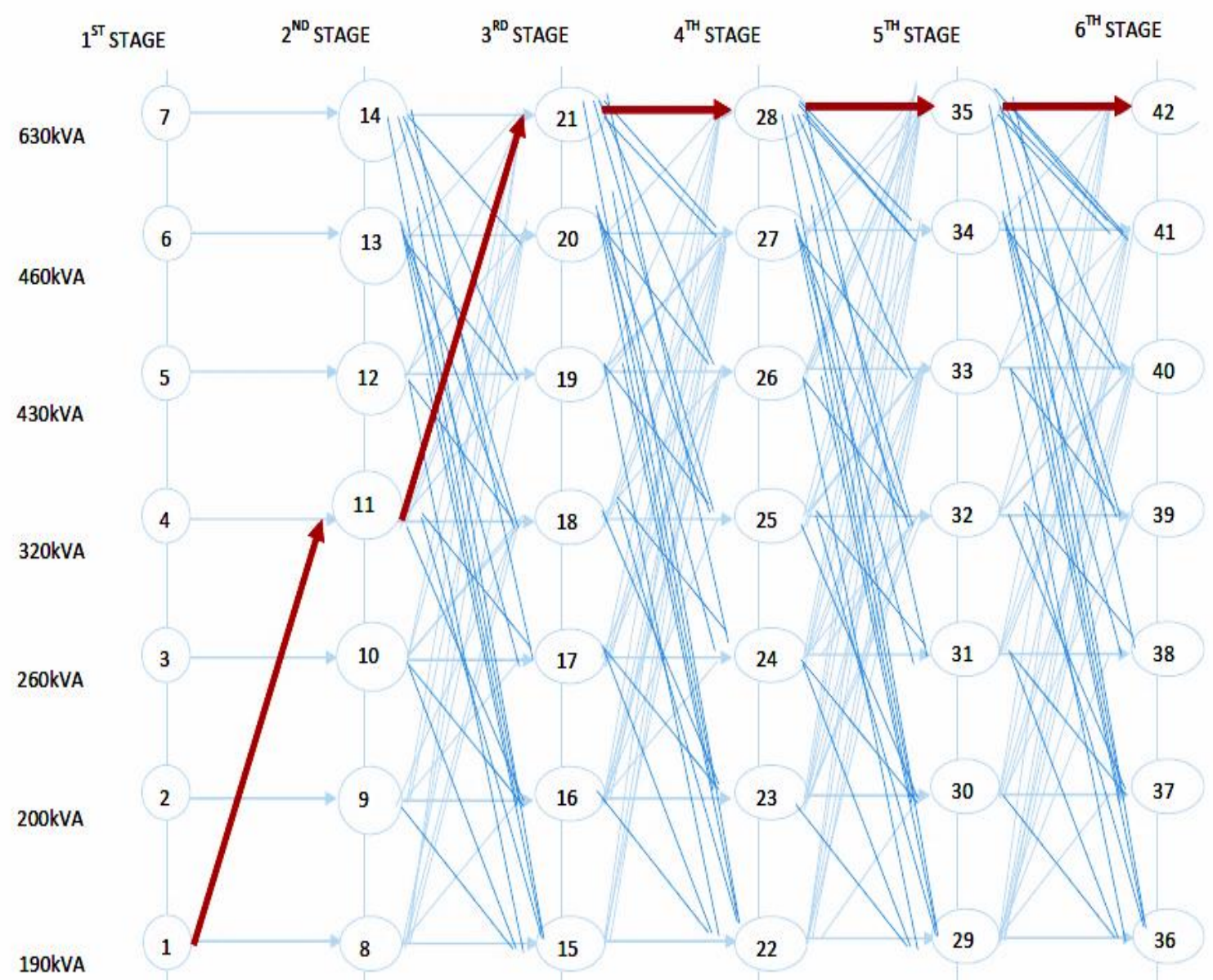

Figure 2:The graph of transformer optimal sizing problem using EAS.

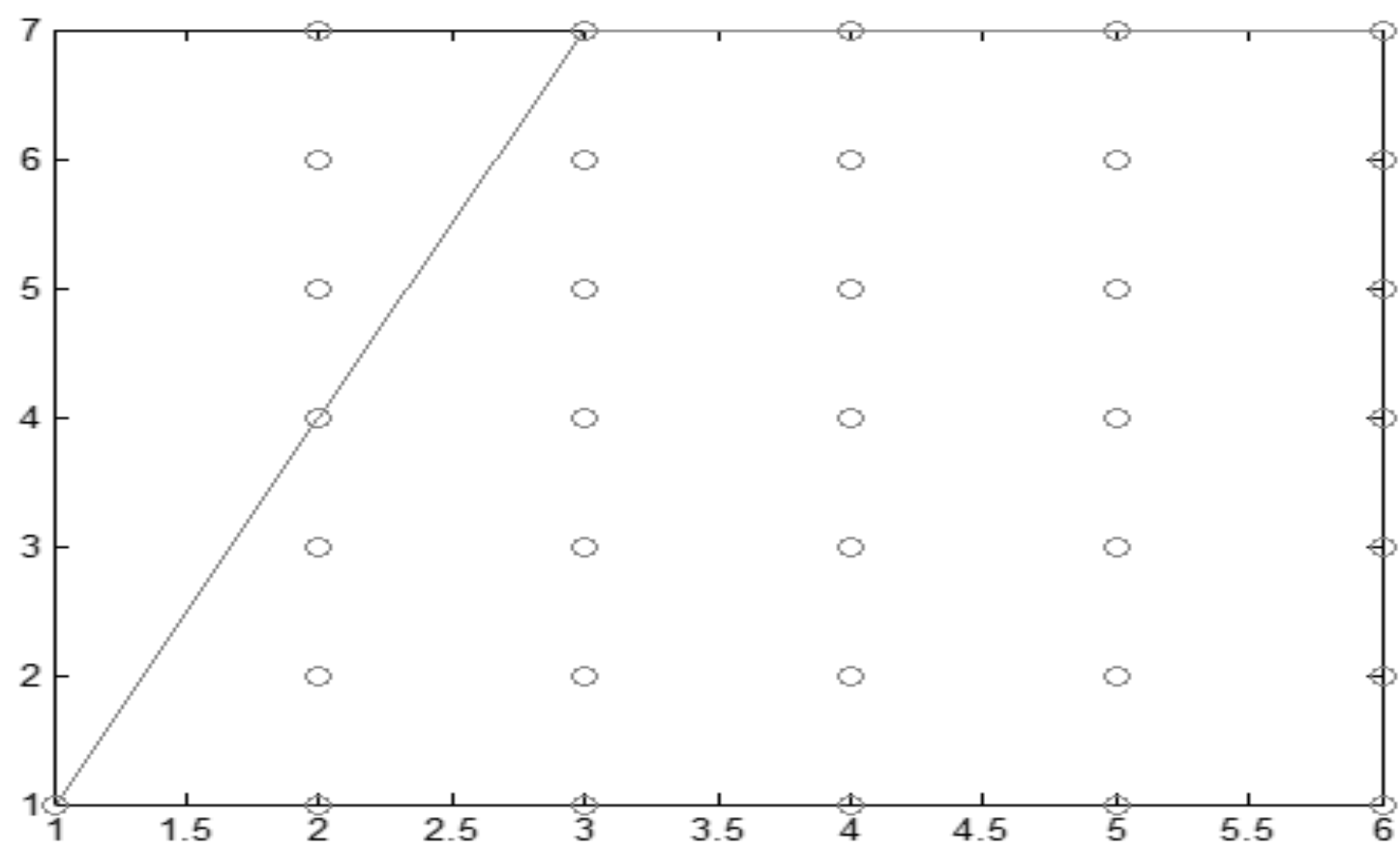

Figure 3: The least cost path derived by the execution of the Matlab code 


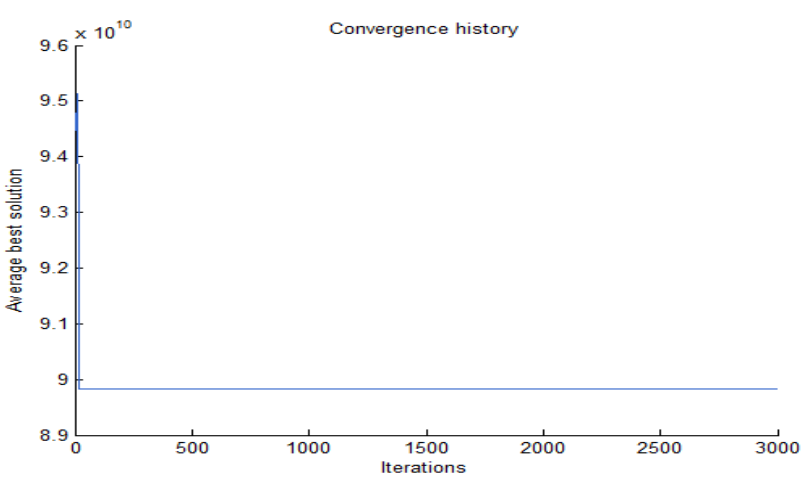

Figure 4: The convergence history graph derived by the execution of the MATLAB code

\section{CONCLUSION}

This study proposes using ant colony optimisation algorithm (an optimisation method) for sizing the distribution transformer(s) of power system. The proposed method accounts for the constraints imposed by the load the transformer serves throughout its lifetime. Consequently, this approach minimises the overall cost of the transformer over its life cycle as well as meets the peak loading condition.

The least cost path and the convergence history graphs were obtained as a result of the execution of the MATLAB code which validated the EAS graph. The algorithm showed a significant saving in capital cost,as the transformer was changed from its initial installed capacity of 190kVA to $320 \mathrm{kVA}$ in the second stage and then finally to 630kVA in the third stage which sufficed for the remainder period under consideration. This finding is in contrast to the traditional simplified sizing strategy usually adopted by utilities companies as shown in the load prediction graph (figure 1).

\section{REFERENCES}

[1] Bonabeau, E. Dorigo, M. and Theraulaz, G., Swarm intelligence from natural to Artificial Systems, Oxford University Press, New York, 1999.

[2] Chapman, S. Electrical Machinery Fundamental, Mc Graw Hill, New York, 2012.
[3] Chen, C. S. and Wu, T. H. "Optimal Distribution Transformer Sizing by Dynamic Programming", Electrical Power \& Energy Systems, Vol. 20, Number 3,pp.161-167, 1998.

[4] Dorigo, M. "Optimisation Learning and Natural Algorithms", Milan, 1992.

[5] Dorigo, M. and Stützle, T. "Ant Colony Optimisation", MIT Press, Cambridge, 2004.

[6] Dorigo, M. Maniezzo, V. and Colorni, A. "Ant System: Optimisation by a Colony of Cooperating Agents," IEEE Transactions on Systems, Man, and Cybernetics, Vol. 26, pp.29-41, 1996.

[7] EC21 global market place, "Technical Parameters", https://www.ec21.com, Accessed on June 20, 2016.

[8] Elefterios, I. Marina, A. Pavlos, S. and Antonios G. "Ant Colony Solution to Optimal Transformer Sizing Problem", 9th International conference on Electrical Power Quality and Utilization, Barcelona, October 9-11, pp.685-690, 2007

[9] Eleftherios, I. and Marina, A. "Ant Colony Solution to the Optimal Transformer Sizing and Efficiency Problem in Power Systems", Nova Science Publishers, 2010, .

[10] Heunis, S. and Herman, R. "A Thermal Loading Guide for Residential Distribution Transformers Based on Time-Variant Current Load Models", IEEE Transaction on Power Systems, Vol. 19, Number 3, pp.1294-1298. , 2004.

[11] Jovanovic, D. "Planning of Optimal Location and Sizes of Distribution Transformers Using Integer Programming", Electrical Power and Energy Systems, vol. 24, pp.717-723, 2003.

[12] Nourelfath, M. and Nahas, N. "An Ant Colony Approach to Redundancy Optimisation for Multistate System", International Conference on Industrial Engineering and Production Management (IEPM 2003), Porto Portugal, pp.6067. , 2003 